\title{
SULLE MANIFESTAZIONI FUMAROLICHE, SOLFATARI- CHE ED IDROTERMALI NEL NW DELLA REPUBBLICA ARGENTINA E LORO POSSIBILITÀ DI UTILIZZAZIONE INDUSTRIALE
}

\section{Giongio Bartolucci}

Nei mesi di aprile e maggio del corrente anno lo scrivente ha avuto occasione di visitare varie aree con manifestazioni fumaroliche ed idrotermali nel N-W del territorio della Repubblica Argentina.

Scopo delle visite era quello di giudicare le eventuali possibilità di sfruttamento dell'eccezionale stato termico (rilevato dalle manifestazioni superficiali) del sottosuolo delle aree in cui le manifestazioni stesse ricadono; sfruttamento realizzabile emungendo dalle profondità i fluidi endogeni caldi, che alimentano le manifestazioni superficiali in quantità ed in condizioni termiche tali da poterli utilizzare per produzione economica di energia elettrica.

Come noto, tale tipo di sfruttamento è da decenni in atto nella cosidetta Regione Boracifera Toscana, ove si emunge dal sottosuolo vapore acqueo surriscaldato con associate piccole quantita di gas vari e di sali, ed è in fase di realizzazione ad Ischia e a Wairakei (Nuova Zelanda, ove si emunge dal sottosuolo acqua calda con disciolte piccole quantità di gas e sali. Anche nel Katanga (Congo Belga) è stato realizzato in questi ultimi anni un impianto che, utilizzando una sorgente di acqua calda a circa $98^{\circ} \mathrm{C}$, fornisce energia elettrica ad una miniera vicina $\left({ }^{1}\right)$.

Panorama geologico e morfologico del N-W argentino ( ${ }^{2}$ ).

La porzione $\mathrm{N}-\mathrm{W}$ dell'Argentina (all'incirca tra i paralleli $22^{\prime \prime}$ e $27^{\circ}$ Sud ed i meridiani $68^{\circ}$ e $63^{\circ}$ Ovest di Greenwich), e precisamente l'area

(1) I fluidi endogeni, qualunque sia il loro stato fisico, oltre che essere sfruttabili per il loro contenuto termico, sono sfruttabili anche per il loro contenuto in gas e sali che, se presenti in quantità sufficienti, possono essi stessi essere oggetto di utilizzazione. Tale sfruttamento però difficilmente può risultare da solo economico ed $\dot{e}$ in generale da prendere in considerazione solo se accoppiato alla utilizzazione termica.

(2) Sono stati consultati principalmente i seguenti lavori: A. Wixomausen, Geologiu Argentina; P. Sckosso, Contribución al conocimiento de la minería y 
coperta dalle provincie di Salta e Jujuy e parte delle provincie di Tucuman e Catamarca, presenta dal punto di vista geologico e morfologico divisioni naturali ben definite.

In essa si distinguono due zone principali, allungate all'incirca per N-S, e precisamente (procedendo da Ovest verso Est): la " Regione Andina Punense " e la «Regione Subandina ».

La "Regione Punense ». - La Regione Andina Punense, o della "Puna di Atacama ", si estende dal confine col Cile ad Ovest fino all'incirca al meridiano $65^{\circ} \mathrm{W}$ ad Est.

La "Puna " è un altopiano, di altezza sul mare di circa 4000 metri, da cui si innalzano varie catene montagnose, allungate in direzione $\mathrm{N}-\mathrm{S}$, che raggiungono anche i 6000 metri circa.

La " Puna " ̀̀ un deserto, con scarsa e povera vegetazione, caratterizzato dalla mancanza di sfogo al mare dei corsi di acqua che scendono dalle sue catene montagnose e che si esauriscono per infiltrazione nelle parti pianeggianti dell'altopiano. In queste si ritrovano anche grandi saline.

Il clima della regione, date la latitudine e l'altitudine, è caratterizzato da una stagione secca invernale fredda (da aprile a novembre) e da una stagione piovosa temperata estiva nel resto dell'anno.

Dal punto di vista geologico la regione della “ Puna » è composta da un basamento di rocce cristalline precambriche, piegate ed erose, sopra cui nel Paleozoico Inferiore si sono depositati sedimenti marini. Poi la regione emerse e di nuovo fu piegata ed erosa, in parte fino al basamento precambrico. Solo nel Cretacico essa fu poi parzialmente invasa dal mare ed infine, a partire dalla fine del Mesozoico, essa è restata emersa fino ai giorni nostri, innalzandosi, nelle diverse fasi dell'orogenesi andina, fino all'attuale altezza sul mare.

Pertanto nel Terziario e nel Quaternario nella regione della « $\mathrm{Pu}$ na ) si sono formati, solo nelle depressioni tra catene montagnose, sedimenti fluviali e lacustri; nell'insieme però la regione è stata (ed è) zona di forte degradazione (specie per deflazione). Estese coltri lavi-

geologíu del noroeste argentino; E. Canton, Aguas minerales del Norte de la Republica Argentina; L. R. Catalano, Datos hidrogeológicos del desierto de Atacama; G. Bonanelli, Tercera contribución al conocimiento de las regiones petroliferas del Norte (Salta y Jujuy); J. RAsmuss, La cuenca de Metan. 
che, dovute alle diverse fasi vulcaniche collegate allorogenesi andina, hanno ricoperto le rocce preesistenti dal Terziario ad oggi.

L'orogenesi andina, iniziatasi nel Terziario Inferiore, si propagò da Ovest verso Est. Perciò le effusioni più antiche, collegate alle grandi dislocazioni che si verificarono all'inizio dell'orogenesi, si ritrovano in Cile, ad Ovest della Puna.

Le dislocazioni tettoniche (e le effusioni ad esse collegate) continuarono per tutto il Terziario, e continuano nel Quaternario, spingendosi man mano verso Est e dando luogo alla serie di enormi " horst ) e "graben" che costituiscono le attuali catene e parti pianeggianti della Puna.

La "Regione Subandina ». - - Il limite Est della regione della Puna è costituito da una serie di faglie, circa per N-S, che in breve tratto abhassano fortemente la zolla orientale. Come conseguenza, l'altopiano, che nella Puna si trova a circa $4000 \mathrm{~m}$ s. m., nella Regione Subandina si trova a poche centinaia di $\mathbf{m}$ s. $m$.

Da questo altopiano si innalzano, con direzione N-S all'incirca, le cosidette "Catene Subandine ), che verso Est si estendono fino alle pianure del Chaco e che raggiungono al massimo circa i $2000 \mathrm{n} \mathrm{s.m.}$

Si tratta di una serie di catene montagnose, relativamente poco elevate, che si estendono tra $\mathrm{i}$ meridiani $65^{\circ} \mathrm{W}$ e $64^{\circ} \mathrm{W}$ dalla Provincia di Tucuman al Sud fino alla Bolivia al Nord.

Le catene subandine sono tutte costituite da un nucleo di rocce del Paleozoico Inferiore (o prepaleozoiche), che affiorano nella parte centrale di esse, e sopra le quali, discordanti, si trovano, sui fianchi delle catene, sedimenti del Cretacico, lagunari o di mare poco profondo, che gradatamente passano in alto ai depositi del cosidetto Terziario Subandino.

Dette catene sono generalmente limitate sia longitudinalmente che trasversalmente da faglie. Nell'insieme quindi esse costituiscono una zolla, allungata per N-S, irregolarmente rialzata.

In sostanza dunque la Regione Subandina si differenzia dalla Regione Punense solo per i molto meno marcati fenomeni di sollevamento e per il fatto di essere stata, dal Terziario Inferiore in poi, in massima parte zona di forte sedimentazione, al contrario della Puna che nello stesso periodo è stata in massima parte zona di forte degradazione.

Le Catene Subandine sono state prodotte dal più orientale piegamento dovuto alla orogenesi andina, che ha ivi agito in tempo relativa- 
mente recente (fine del Terziario) ed in maniera più attenuata clie non nella regione della Puna, senza che si siano verificate effusioni o eruzioni in genere. Nelle Catene Subandine non si ritrova infatti alcun apparato vulcanico.

Il clima della regione subandina è in genere subtropicale con stagione estiva piovosa e stagione invernale secca.

\section{Caratteristiche geologiche del NW argentino.}

Le formazioni delle regioni "Punense " e "Subandina", come già precedentemente detto, appartengono a varie età dal Precambrico all'Attuale.

La serie precambrica è costituita da scisti quarzitici, scisti cloritici e filladi, costantemente con direzione $N-S$ e forte immersione.

I sedimenti paleozoici (Cambrico Superiore, Silurico e Devonico), che con marcata discordanza seguono in alto la serie precambrica, sono costituiti da conglomerati basali, arenarie e quarziti, scisti argillosomicacei, filladi (con intercalazioni calcaree fossilifere) e poi nuovamente arenarie quarzose e micacee. Naturalmente non tutta la serie è sempre localmente presente. La serie paleozoica si presenta nei vari luoghi da orizzontale fino a fortemente inclinata, sempre però con direzione all'incirca $N$-S. Lo spessore complessivo di questa serie è di varie migliaia di metri.

La serie postpaleozoica presente nel $N W$ argentino può dividersi come segue: $a$ ) arenarie inferiori; $b$ ) orizzonte calcareo-dolomitico con Melania; $c$ ) marne multicolori; $d$ ) arenarie superiori; $e$ ) terziario subandino; $f$ ) strati jujegni e della Puna; g) quaternario. I termini inferiori della serie sono anche chiamati " serie petrolifera", dato che nel Nord della Provincia di Salta in essi è stato ritrovato petrolio.

a) Delle " arenarie inferiori ) è detta la formazione che costituisce la parte inferiore della serie postpaleozoica. Detta formazione in generale inizia in basso con un conglomerato basale, non sempre presente e di spessore vario. Seguono, predominando, arenarie quarzose con, nella parte superiore, intercalazioni marnose. Lo spessore della formazione è molto variabile: può mancare e può superarc i 2000 metri. Queste forti variazioni sono interpretate come dovute all'esistenza di un antico rilievo, presente durante la deposizione.

In genere le arenarie inferiori sono poco discordanti rispetto alla formazione paleozoica su cui poggiano e sembrano essere di formazione in parte fluviale ed in parte di mare poco profondo. 
L'opinione più diffusa è che, come età, esse siano del Cretacico Inferiore. In ogni caso esse sono sicuramente preterziarie essendo state interessate dai movimenti di questa era.

b) L'orizzonte "calcareo-dolomitico con Melania " è superiore, ed in generale concordante, con quello delle arenarie inferiori. Esso è costituito da arenarie calcaree, calcari dolomitici (predominanti) e poche marne ed argille. Il suo spessore è molto variabile: a volte manca, a volte supera i 200 metri.

Le rocce che lo compongono sono di formazione in parte di acqua dolce e salmastra ed in parte marina di mare poco profondo, e sono generalmente attribuite al Cretacico Medio. Sono state interessate dagli eventi tettonici del Terziario e si presentano molto fratturate.

c) L'orizzonte del calcare dolomitico passa superiormente, gradualmente, ad un complesso, detto delle "marne multicolori", costituito principalmente da argille, marne e marne arenacee, e con alternati banchi di calcare e, nella parte superiore, di arenarie calcaree. Detto complesso lo si divide (dal basso all'alto) in: marne rosse inferiori, marne verdi, marne rosse superiori.

In queste rocce si trovano resti fossili di pesci, di rettili, di piante e di insetti; sembra trattarsi di depositi di acqua dolce. L'età sembra essere eocenico-cretacica. La potenza totale del complesso varia tra 100 e 300 metri.

d) Concordanti con le marne multicolori e ad esse sovrastanti sono le cosiddette "arenarie superiori ). Si tratta di una serie di arenarie, in generale calcaree, con intercalazioni argillose. Lo spessore della serie, di età oligocenica, e che sembra essere in parte fluviale ed in parte marina, è molto variabile.

e) La serie delle arenarie superiori verso l'alto passa gradatamente al "Terziario Subandino", potente serie fluviale, lagunare e lacustre costituita da arenarie (predominanti), argille marnose e marne in parte gessose (con frequenti cambi di facies sia in senso orizzontale che in senso verticale), di età mio-pliocenica.

Il Terziario Subandino è stato affettato dai movimenti terziari. Il suo spessore sembra aumentare verso Nord. Nel Sud della Provincia di Salta (S. Barbara) lo spessore del Terziario Subandino è di circa 400 metri.

f) Gli "strati jujegni" si trovano superiormente al Terziario Subandino nelle Catene Subandine. Si tratta di depositi fluviali del 
Pliocene, non molto dislocati dagli ultimi movimenti dell'orogenesi andina.

Gli «strati della Puna » sono i corrispondenti degli strati jujegni che si trovano nella Regione Punense.

La potenza delle formazioni suddette può anche essere di centinaia di metri.

g) Il "Quaternario » è rappresentato nella Regione Punense da depositi morenici e da formazioni saline (gessi, salgemma, borati) e nella Regione Subandina da depositi lacustri e palustri. Ovunque poi si trovano depositi eolici, spesso anche molto potenti.

Rocce intrusive granitiche e granodioritiche sono presenti, in affioramenti allungati per $N$-S, nella Regione Punense. L'età di queste intrusioni è incerta; in ogni caso però paleozoica o anteriore.

Rocce vulcaniche ricoprono poi vaste estensioni nella regione della Puna. Si tratta di vaste coltri di daciti e lipariti (lave e tufi corrispondenti). Le eruzioni ebbero il loro apogeo nel Miocene, ma continuarono fino al Pleistocene.

Inoltre sono numerosi nella Regione Punense vulcani quaternari basaltici, le cui effusioni ricoprono però aree molto ristrette; di essi alcuni sono stati ancora attivi in tempi storici.

\section{Caratteristiche delle manifestazioni visitate.}

Le manifestazioni visitate nel territorio della Repubblica Argentina si possono dividere in due gruppi ben distinti tra loro: manife. stazioni ricadenti a distanze di pochi chilometri (al massimo 10-15) da vulcani quaternari $\left(^{3}\right)$ e quindi facilmente collegabili a fenomeni magmatici; e manifestazioni non palesemente collegabili a fenomeni magmatici noti perché ricadenti in aree intorno alle quali per vasto raggio (più di un centinaio di chilometri) non esistono apparati o prodotti solidi vulcanici.

Le manifestazioni appartenenti al primo dei suddetti gruppi ricadono tutte nella regione punense andina $\left({ }^{4}\right)$, ad altitudini sul mare

(3) Queste manifestazioni sono da collegare, più o meno direttamente, ai po. stumi di attività di vulcani vicini, pur non potendosi però con sicurezza escludere che $i$ vulcani stessi siano in fase di quiescenza.

(4) Sono state visitate nella regione punense quattro località con manifestazioni termali, e precisamente (vedi cartina annessa): (1) Termas di Pompeya 
di circa 4000 metri, in zone con climi aspri, pressoché disabitate, in cui le vie di comunicazione, quando esistono, sono difficili e spesso interrotte durante la stagione piovosa estiva e che, in generale, non offrono alcuna risorsa per un raggio di centinaia di chilometri.

Dette manifestazioni si sviluppano tutte in zone interessate da faglie (direttamente osservabili sul terreno), in formazioni, permeabili alle acque superficiali, di tufi e lave, e depositi fluviali del Terziario, soprastanti in genere a formazioni più antiche (paleozoiche e precambriche) anche esse facilmente permeabili alle acque.

Le manifestazioni consistono in sorgenti ed affioramenti di acque calde, al massimo a temperatura di ebollizione, ed in esalazioni di vapore proveniente dall'ebollizione o dall'evaporazione delle acque stesse. Si nota la presenza in tutte le manifestazioni di $\mathrm{CO}_{2}$ e di $\mathrm{H}_{2} \mathrm{~S}$.

Le manifestazioni sono senz'altro da ritenere secondarie: le acque calde, anche bollenti, affioranti sono cioè un miscuglio di fluidi endogeni caldi risalienti dalle profondità con acque fredde di provenienza meteorica. Il miscuglio avviene sicuramente fin da considerevoli profondità, sia pure con acque superficiali provenienti da zone di infiltrazione lontana, dato che nella serie dei terreni della Regione Punense mancano estese formazioni impermeabili che possono costituire una efficace protezione contro l'infiltrazione delle acque superficiali (la serie cretacica marina, nei luoghi ove si depositò, è stata in gran parte erosa).

Le manifestazioni del secondo gruppo ricadono invece tutte nella cosidetta Regione Subandina ("), ad altitudini sul mare inferiori ai 1000

(2) Baños de Tocomar, (3) Baños de Incachuli, (4) Baños del Tuzgle.

Le suddette località non sono tutte quelle in cui, nella regione punense, si hanno manifestazioni termali (sono state visitate solo quelle che $\dot{e}$ stato possibile raggiungere con relativa facilità); ne esistono molte altre le cui manifestazioni yerò, dalle notizie raccolte, sembra si presentino tutte con le stesse caratteristiche intrinseche ed estrinseche delle manifestazioni visitate.

(5) Sono state visitate nella regione subandina le seguenti località con manitestazioni termali (vedi cartina annessa): (5) Termas de Rosario de la Frontera, (6) Baños del Galpón, (7) Baños des Los Sances, (8) Termas del Palmar, (9) Termas de la Quinta, (10) Siete Aguas, (11) Aguas Calientes de Caimancito.

Altre località con manifestazioni termali, esistenti nella regione, non si sono potute visitare per mancanza di sufficienti dati sulle loro ubicazioni. Non sembra però che ivi le manifestazioni siano più vistose di quelle visitate. 
metri, in zone relativamente popolose, che godono di buon clima, con buone vie di comunicazione, e che, in generale, offrono discrete risorse.

Queste manifestazioni si rinvengono tutte sulle pendici delle Catene Subandine, alcune nei terreni impermeabili della serie delle " marne multicolori ), e in terreni a queste stratigraficamente superiori, altre invece nei terreni permeabili inferiori.

Anche queste manifestazioni consistono in sorgenti ed affioramenti di acque calde, al massimo a temperatura di ebollizione, ed in esalazioni di vapore proveniente dalla ebollizione e dall'evaporazione delle acque stesse, ed in esse si nota in genere la presenza di $\mathrm{CO}_{2}$ e di $H_{2} S$.

Anche queste manifestazioni hanno sede in zone interessate da faglie direttamente osservabili sul terreno $e$, in alcuni casi, con rigetti imponenti.

Le manifestazioni nei terreni permeabili inferiori sono da ritenere senz'altro secondarie. Per quelle dei terreni impermeabili superiori, affioranti all'intorno di esse per vaste estensioni, non si può escludere invece un'origine primaria; comunque esse deblono essere poco influenzate da acque di provenienza meteorica; se acque meteoriche intervengono esse debhono essere di infiltrazione lontana.

\section{Considerazioni.}

L'esperienza fin qui acquisita e gli studi eseguiti nel campo dei fluidi endogeni caldi permettono di poter con sicurezza assumere che l'origine di essi è profonda e connessa, più o meno direttamente, con la presenza di magma, o comunque di masse fuse a temperatura elevata, nel sottosuolo.

Inoltre nelle aree ove fluidi endogeni caldi esistenti nel sottosuolo sono stati captati in condizioni ed in quantità interessanti si è osservato che:

a) esistono nei terreni importanti soluzioni di continuità (fratture con o senza rigetto), e cioè zone di minor resistenza al moto dei fluidi, che quindi rappresentano vie di raccolta e di facile ascesa dei fluidi verso la superficie.

b) esistono nella serie geologica dei terreni, a profondità economicamente possibile, formazioni lapidee permeabili in grande atte 
al contenimento e ad una facile circolazione di fluidi, e cioè favorevoli ad un relativamente facile ritrovamento di essi.

Oltre le due suddette condizioni, nella Regione Boracifera Toscana ne esiste una terza e cioè:

c) è presente nella serie geologica dei terreni una formazione superiore nell'insieme impermeabile, estesa, sufficientemente potente e tettonicamente cosi disposta che, fra l'altro, impedendo l'infiltrazione nel sottosuolo delle acque meteoriche fredde, non permette la degradazione termica del fluido endogeno e dell'ambiente tutto.

Si ha dunque come dato di fatto che nella Regione Boracifera Toscana, ove manifestazioni superficiali rivelano un eccezionale stato termico del sottosuolo, si riscontrano tutte e tre le condizioni suddette e si emunge dal sottosuolo vapore acqueo surriscaldato.

A Wairakei ed a Ischia, ove pure un eccezionale stato termico del sottosuolo è rivelato da manifestazioni superficiali, si riscontrano solo le prime due condizioni di cui sopra, e si emunge dal sottosuolo finora raggiunto con le perforazioni acqua calda sotto pressione.

Prescindendo dunque dal problema della origine prima del fluido endogeno, dal suo stato fisico nel sottosuolo e dai fenomeni che possono interessarlo durante la sua venuta a giorno, in ogni caso le condizioni suelencate sono da ritenere per lo meno condizioni favorevoli alla possibilità di ritrovare nel sottosuolo fluidi endogeni economicamente sfruttabili in quelle aree in cui l'esistenza di manifestazioni superficiali rivela l'eccezionale stato termico del sottosuolo.

Nello specchietto che segue sono riportate le caratteristiche dei due gruppi di manifestazioni visitate nel territorio argentino, sia per quanto riguarda le condizioni favorevoli al ritrovamento economico di fluido caldo nel sottosuolo, precedentemente elencate, che per quanto riguarda altre condizioni di importanza pratica.

Dallo specchietto si vede che, per quanto riguarda le condizioni generali, le manifestazioni del secondo gruppo sono molto più favorevolmente indiziate dal punto di vista minerario-industriale.

Inoltre, solo per alcune delle aree con manifestazioni del secondo gruppo, sono presenti tutte e tre le condizioni precedentemente elencate come favorevoli al ritrovamento economico di fluido caldo nel sottosuolo.

Tale tipo di aree è pertanto da considerare il più favorevolmente indiziato (tra quelli considerati) per una ricerca mineraria per " Forze Endogene ". 


\begin{tabular}{|c|c|c|}
\hline Condizioni & $\begin{array}{c}\text { lo gruppo } \\
\text { (manifestazioni fa- } \\
\text { cilmente collegabi- } \\
\text { li a vulcani quater- } \\
\text { nari) } \\
\text { Regione Punense }\end{array}$ & $\begin{array}{c}2^{\circ} \text { gruppo } \\
\text { (manifestazioni non } \\
\text { collegabili } \\
\text { a vulcanismo) } \\
\text { Regione Subandina }\end{array}$ \\
\hline Esistenza di faglie & Si & Si \\
\hline $\begin{array}{l}\text { Esistenza nella serie dei terreni di for- } \\
\text { mazioni superiori impermeabili }\end{array}$ & No & $\begin{array}{l}\text { Si } \\
\text { (per alcune delle } \\
\text { manifestazioni) }\end{array}$ \\
\hline $\begin{array}{l}\text { Esistenza nella serie dei terreni di for- } \\
\text { mazioni lapidee permeabili in gran- } \\
\text { de a profondita economica }\end{array}$ & Si & Si \\
\hline $\begin{array}{l}\text { Temperatura massima del fluido delle } \\
\text { manifestazioni superficiali }\end{array}$ & Ebollizione & Ebollizione \\
\hline $\begin{array}{l}\text { Condizioni geografiche generali delle } \\
\text { aree nelle quali le manifestazioni ri- } \\
\text { cadono (clima, comunicazioni, popo- } \\
\text { lazione, risorse, ecc.). }\end{array}$ & Pessime & Discrete \\
\hline
\end{tabular}

Allo stato attuale delle conoscenze non è possibile una basata previsione sullo stato fisico del fluido che si potrà in caso emungere dal sottosuolo: non è possibile cioè prevedere con sicurezza se si emungerà vapore (possibilmente surriscaldato) o acque calde.

Il parallelo con la Regione Boracifera Toscana però conforta la speranza che si possa emungere vapore surriscaldato; in ogni caso, è sicuro che la temperatura del fluido che si emungerà dal sottosuolo sarà superiore a quella del fluido delle manifestazioni, e cioè superiore alla temperatura di ebollizione alla pressione atmosferica.

Date le discrete condizioni geografiche generali di cui godono le aree in parola, anche nel caso relativamente sfavorevole che il fluido che si emungerà sia acqua calda, nella giustificata previsione di una temperatura crescente con la profondità, una utilizzazione industriale di esso non appare di difficile attuazione.

Centro Studi "Larderello " - Centro di Studio per la Geologia Tecnica del C.N.R. presso l'Istituto di Geologia Applicata e di Giacimenti Minerari della Università (Facoltà di Ingegneria), Roma (San Pietro in Vincoli) - Ottobre 1955.

\section{RIASSUNTO}

Si descrivono diverse manifestazioni esulativo-idrotermali visitate, nella primavera del 1955. nelle Provincie di Salta e Jujuy (Repubblica Argentina). 
Le manifestazioni vengono suddivise in due gruppi secondo che esse sono o no palesemente collegabili a fenomeni magmatici noti nelle loro vicinanze.

Sulla base delle caratteristiche geologiche e geografiche delle aree in cui le manifestazioni si rinvengono e sulla base delle caratteristiche intrinseche delle manifestazioni stesse, si considerano le possibilità di utilizzazione industriale dello stato termico del sottosuolo delle aree in parola.

Si conclude che, tra quelle considerate, le aree più favorevolmente indiziate sono quelle in cui ricadono manifestazioni non direttamente collegabili a fenomeni magmatici noti.

\section{SUMMARY}

Several exhalative-hydrotermal showings are here described which occur in the provinces of Salta and Jujuy (Argentina Republic); these showings were visited in the spring of 1955.

The showings are divided into two groups, depending upon the existance, in their neighbourhood, of quaternary volcanoes which the sane showings can be obviously related to.

The possibilities of industrial utilisation of the underground thermality of the areas are examined on the basis of the geological and geographical characteristics of the areas as well as on the basis of the intrinsical characteristics of the phenomena themselves.

As a conclusion, among those here examined, the areas whose exhalative-hydrotermal phenomena are not directly connectable to known magmatic phenomena appear to be more favourable.

\section{RESUMEN}

Se decriben las caracteristicas de varias manifestaciones exhalativohidrotermales visitadas en las Provincias de Salta y Jujuy (territorio de la Repüblica Argentina) y se subdividen las mismas en dos grupos segün que estén o no visiblemente relacionadas con los fenómenos magmáticos observados en su vecindad.

En base a las características geológicas y geográficas de las áreas donde se hallan las manifestaciones, $y$ a las caracteristicas intrinsecas de las mismas, se considera después la posibilidad de utilización in- 



\section{MANIFESTAZIOMI FUMADOUICHE, SOIFATARIIHE ED IDPOTERMALI VISITAIE NEL NW DELLA}

REPUBBLLCA ARGEMTINA

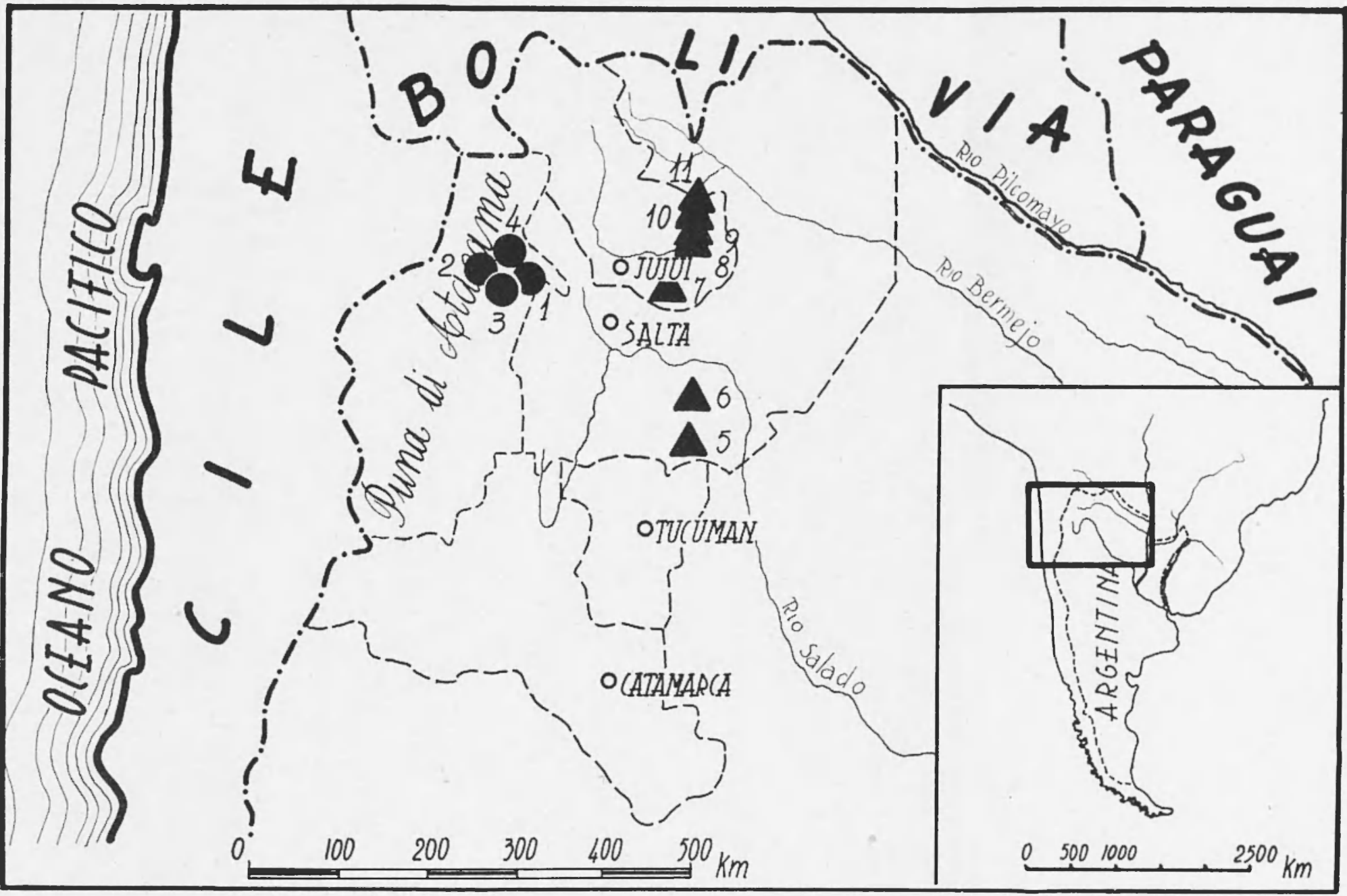

- 10Gruppo di manifestazioni

1. TIRMAS DE POMPEYA

2. BAÑOS DE TOCOMAR

3. BAÑOS DE INCACHULI

4. BAÑOS DE TUZGLI
$\Delta 2^{\circ}$ Gruppo di manifestazioni

5 . TARMAS DE ROSARIO de LA FRONTERA 9_ TERMAS IE LA QUIIITA 6 - BAÑOS DE GALDÓN

7 - BAÑOS DE lOS SAUCES

8 . TIRMAS DE DALMAR
10_SIETE AGUAS

11 . AGUAS (ALIEMTES

OE CAIMANCITO 